\title{
PRÁCTICAS DE ENSEÑANZA PARA LA INCLUSIÓN DE ESTUDIANTES CON DISCAPACIDAD INTELECTUAL EN ARGENTINA DURANTE TIEMPOS DE COVID-19
}

\section{Teaching Practices for the Inclusion of Students with Intellectual Disabilities in Argentina during Times of COVID-19}

María Eugenia YADAROLA Universidad Católica de Córdoba m.eugenia.yadarola@gmail.com

Carolina del Valle Erramouspe Universidad Católica de Córdoba

Federici Tomás GonzÁlez

Universidad Católica de Córdoba

Vanesa Nelly LAmbertucci

Universidad Católica de Córdoba

Karina Elizabeth Medina

Universidad Católica de Córdoba

María Laura Molina

Universidad Católica de Córdoba

Carolina Andrea Sesto

Universidad Católica de Córdoba

Romina Soledad VALDEZ

Universidad Católica de Córdoba

Los/as autores/as conforman la Línea de investigación "Inclusión Educativa de personas con discapacidad: Políticas y prácticas” de la Universidad Católica de Córdoba como Unidad Asociada al CONICET 


\section{PRÁCTICAS DE ENSEÑANZA PARA LA INCLUSIÓN DE ESTUDIANTES \\ CON DISCAPACIDAD INTELECTUAL EN ARGENTINA DURANTE TIEMPOS DE COVID-I9 \\ M. E. YADAROLA, C. DEL VALLE ERRAMOUSPE, F. TOMÁs GONZÁLEZ...}

Recepción: 15 de octubre de 2020

Aceptación: 22 de diciembre de 2020

Resumen: El objetivo del estudio fue indagar si las prácticas de enseñanza desarrolladas en tiempos de aislamiento social preventivo obligatorio en Argentina favorecen la inclusión, los aprendizajes y la autonomía de los/as estudiantes con discapacidad intelectual, en opinión de sus profesionales de apoyo a la inclusión y de sus familias. Se realizó un diseño exploratorio-descriptivo, con un muestreo intencional. Se aplicó un cuestionario semiestructurado a 75 profesionales de apoyo a la inclusión y a 40 familias de niños/as y adolescentes con discapacidad intelectual incluidos en escuelas comunes de gestión estatal y privada de Córdoba (mayoritariamente) y otras provincias argentinas. Como resultado el Estado no ha logrado garantizar el acceso de sus estudiantes con discapacidad intelectual, en igualdad de condiciones, a la enseñanza remota de emergencia no solo por no asegurar la tecnología necesaria y la conectividad. La tarea docente se centró en planificaciones homogéneas dejando a los/as profesionales de apoyo externos las adaptaciones curriculares, la atención directa con el/ la estudiante con discapacidad y el asesoramiento a las familias. El vínculo del docente con el/la estudiante con discapacidad intelectual muestra escasa comunicación, escucha y relación positiva. Como consecuencia muchos/as estudiantes no han alcanzado los objetivos de aprendizaje propuestos ni la autonomía esperada.

Palabras Clave: inclusión educativa; discapacidad intelectual; COVID-19; prácticas de enseñanza; enseñanza remota de emergencia.

AвsтRACт: The objective of the study was to investigate whether the teaching practices developed in times of compulsory preventive social isolation in Argentina favor the inclusion, learning and autonomy of students with intellectual disabilities, in the opinion of their professionals supporting inclusion and of their families. An exploratory-descriptive design was carried out, with an intentional sampling. A semi-structured questionnaire was applied to 75 inclusion support professionals and $40 \mathrm{fam}-$ ilies of children and adolescents with intellectual disabilities included in common schools of state and private management, in Córdoba (mainly) and other Argentine provinces. As a result, the State has not been able to guarantee access of its students with intellectual disabilities, under equal conditions, to emergency remote teaching not only for not ensuring the necessary technology and connectivity. The teaching task focused on homogeneous planning, leaving the curricular adaptations to the external support professionals, direct attention to the student with disabilities, and counseling for families. The teacher"s relationship with the student with intellectual disability shows poor communication, listening and a positive relationship. Consequently, many students have not reached the proposed learning objectives nor the expected autonomy.

KEYwORDS: educational inclusion; intellectual disability; COVID-19; teaching practices; remote emergency teaching.

(Consejo Nacional de Investigaciones Científicas y Técnicas de Argentina). La Dra. Yadarola es la directora de la Línea. 
ATENCIÓN TEMPRANA EN TIEMPOS DE COVID-I9: INVESTIGAR LA/S REALIDAD/ES DE LA TELEINTERVENCIÓN EN LAS PRÁCTICAS CENTRADAS EN LA FAMILIA M. E. YADAROLA, C. DEL VALLE ERRAMOUSPE, F. TOMÁs GONZÁLEZ...

\section{Introducción}

L

A SITUACIÓN INÉDITA PROVOCADA POR EL COVID-I9 ha impactado económica, cultural y socialmente en todos los países del mundo. Cierre de fronteras, prohibición para viajar de un país a otro y aislamiento preventivo fueron algunas medidas adoptadas con el fin de combatir la propagación de la pandemia y el contagio masivo de la población.

Es importante hacer un análisis de la realidad en la República Argentina, sobre todo del contexto donde se da esta situación extraordinaria. Es en este marco que nos interesó indagar, como objetivo del estudio, si las prácticas de enseñanza desarrolladas en tiempos de aislamiento social preventivo obligatorio en Argentina favorecen la inclusión, los aprendizajes y la autonomía de los/as estudiantes con discapacidad intelectual, en opinión de sus profesionales de apoyo a la inclusión y de sus familias.

Primeramente, en este apartado, contextualizamos la situación en Argentina como respuesta al COVID-19, focalizando en algunas consecuencias del aislamiento social preventivo obligatorio. Luego, mencionamos los marcos legales en Argentina para la inclusión educativa de las personas con discapacidad, analizándolos desde marcos teórico-conceptuales que dan fundamento a la interpretación de los datos recolectados para este estudio.

El Gobierno Nacional anunció el aislamiento social preventivo obligatorio tempranamente (20 de marzo de 2020), como una medida excepcional en este contexto crítico, ubicando a nuestro país como uno de los primeros Estados en tomar esta medida; pero también, a esta altura, lo posiciona por contar con una de las cuarentenas más largas del mundo. El propósito expresado fue reducir el contacto para minimizar las posibilidades de contagios, con relativos logros al respecto, pero con un gran impacto socioeconómico, educativo y emocional adicional generado por el aislamiento.

El ámbito educativo no estuvo exento de este cambio súbito e impacto, porque en pocas semanas pasó de las clases presenciales en contacto directo con grupos de pares y docentes, a la implementación de prácticas educativas virtuales, sincrónicas o asincrónicas, o prácticas remotas con otros formatos, según la elección de cada establecimiento educativo y/o docente.

Según los datos publicados en el mes de marzo por la Unesco (2020) están afectados 363 millones de estudiantes en el mundo desde el nivel preescolar hasta el nivel superior. Como consecuencia, a nivel mundial en el mes de marzo, 1 de cada 5 estudiantes de primaria y secundaria no asistían a clases en la escuela y 1 de cada 4 no asistía a la enseñanza superior.

La realidad educativa en Argentina, y por motivo del confinamiento preventivo, dispuesto a pocos días de iniciado el ciclo lectivo, llevó al cierre de todos los establecimientos educativos (nivel inicial, primario, secundario y superior) sin asistencia presencial, utilizando la enseñanza remota de emergencia como medio para dar continuidad a las clases. Entendemos por enseñanza remota de emergencia, en tanto acordamos que "A diferencia de las experiencias planificadas desde el principio y diseñadas para 
estar en línea, la enseñanza remota de emergencia (ERT) es un cambio temporal de la entrega de instrucción a un modo de entrega alternativo debido a circunstancias de crisis. Implica el uso de soluciones de enseñanza totalmente remotas para la instrucción o la educación que de otro modo se impartirían presencialmente o como cursos combinados o híbridos y que volverán a ese formato una vez que la crisis o la emergencia hayan disminuido. (Hodget et al., 2020, p. 1)" La enseñanza remota de emergencia puede ser virtual o no.

En Argentina se dispuso la suspensión de actividades académicas presenciales desde el 16 de marzo y por el transcurso de 15 días, pero no volviendo hasta el momento a ser habilitada la presencialidad, a excepción de contadas localidades del país (por lo menos hasta mediados de octubre, fecha de elaboración de este artículo) y avizorando un futuro incierto.

El Gobierno Nacional argentino presentó en septiembre los resultados preliminares de la Evaluación Nacional del Proceso de Continuidad Pedagógica (2020). La encuesta realizada a docentes argentinos indicó que la conectividad y el equipamiento tecnológico son las principales dificultades de los estudiantes para seguir la propuesta educativa; también se señalan las dificultades socioeconómicas de los hogares. El informe mostró que el medio de comunicación más utilizado desde el nivel inicial al secundario fue el teléfono celular. En el nivel secundario el $59 \%$ de los/las docentes utilizaron plataformas educativas como Google Classroom o Moodle. Cabe destacar que, según el informe, el $24 \%$ de las/los docentes tuvo menos de un contacto semanal con sus estudiantes (Gobierno de la Nación Argentina, 2020).

Recursos tecnológicos, accesibilidad y disponibilidad de apoyo familiar significativos para llevar adelante el proceso educativo virtual son al menos algunas de las problemáticas que se suman, las cuales interfieren con el acceso y la formación educativa equitativa para los/as estudiantes en general, especialmente en los niveles primario y secundario.

En este contexto, la escuela ha debido adaptarse a estos nuevos escenarios que la pandemia generó y, más específicamente, que el aislamiento social preventivo obligatorio provocó. Dussel (2020, p. 1) afirma que:

La escuela ya no opera principalmente en un espacio físico, pero puede jugar un papel relevante afirmándose como un espacio de conocimiento, un centro de solidaridad pública, y ofreciendo un tiempo que conecta con horizontes de largo plazo, más necesarios que nunca en una situación en la que la urgencia se impone con mucho dramatismo.

En este contexto, se hizo evidente cómo la escuela, buscando garantizar la continuidad pedagógica, tuvo que adaptarse a una dinámica diferente a la que hasta hoy estaba acostumbrada, a utilizar herramientas tecnológicas (simples o complejas) para poder alcanzar a todos los/as estudiantes, como también implementar otros medios no tecnológicos para aquellos/as estudiantes que debido a condiciones más vulnerables necesitan de otras formas para acceder a esta enseñanza remota de emergencia.

Ahora bien, esta enseñanza ya no puede ser abordada de manera circunstancial, es decir, como consecuencia del aislamiento social preventivo obligatorio. Cabe interrogarse sobre los nuevos modos de enseñar y de aprender, de promover la inclusión en un escenario de gran complejidad. 
ATENCIÓN TEMPRANA EN TIEMPOS DE COVID-I9: INVESTIGAR LA/S REALIDAD/ES DE LA TELEINTERVENCIÓN EN LAS PRÁCTICAS CENTRADAS EN LA FAMILIA M. E. YADAROLA, C. DEL VALLE ERRAMOUSPE, F. TOMÁs GONZÁLEZ...

La enseñanza remota de emergencia es el modo o formato que nos permite continuar con las clases en todos los niveles educativos y muchas de las herramientas y modalidades adquiridas hoy permanecerán en el sistema educativo postpandemia.

Las construcciones abordadas desde esta perspectiva contemplan prácticas pedagógicas variadas, singulares y enriquecidas destinadas a cada uno/a de los/as estudiantes basadas en una educación personalizada, porque los/as estudiantes aprenden de manera diferente y a ritmos distintos. Hoy esta mirada se torna imprescindible, creando estrategias que faciliten el aprendizaje de todos/as los/as estudiantes desde sus hogares, ofreciendo un abanico de mediaciones, de posibilidades y estímulos, en especial a aquellos/as más vulnerables, permitiendo a cada estudiante construir su propio desarrollo.

Varias de las situaciones que la pandemia ha puesto de manifiesto con respecto a las personas con discapacidad y/o poblaciones vulnerables no son nuevas, simplemente se hicieron visibles. Como menciona Alasuutari (2020, p. 1):

Quienes eran excluidos antes de la pandemia presentan ahora mayor riesgo. La interseccionalidad entre pobreza, género, etnia, edad, discapacidad y otras identidades puede resultar en múltiples maneras de discriminación y exclusión. Es por eso que los esfuerzos en salud, protección social, educación y otros sectores deben tomar en cuenta a todos al momento de afrontar la pandemia con el fin de garantizar la continuidad de servicios seguros e inclusivos para todos.

Los riesgos específicos del aislamiento social preventivo obligatorio para los/as estudiantes en situación de vulnerabilidad, sobre todo para aquellos con alguna discapacidad, "han sido, básicamente, pasados por alto" (Alasuutari, 2020, p. 1).

Ahora bien, en Argentina la inclusión educativa de las personas con discapacidad es un derecho reconocido en sus marcos legales, por lo que debe ser garantizada.

En el año 2008, la República Argentina adhiere y ratifica la Convención de los Derechos de las Personas con Discapacidad-CDPD-(ONU, 2006), tanto su protocolo facultativo como la Convención, dándole jerarquía constitucional en el país en el año 2014, bajo la Ley n. ${ }^{\circ} 27.044$.

Desde su promulgación en el país, diferentes organizaciones de la sociedad civil como diversos referentes del colectivo de la discapacidad han trabajado insistentemente en el cumplimiento de diferentes derechos como el de la educación inclusiva, accesibilidad, inclusión laboral, etc., que en nuestro país aún sigue siendo materia pendiente en muchos sectores de la sociedad.

En el Programa de las Naciones Unidas para el Desarrollo (PNUD) de la ONU (2015), se plantean 17 Objetivos de Desarrollo Sostenible (ODS) 2015-2030. En el ODS n. ${ }^{\circ} 4$ se propone como finalidad "garantizar una educación inclusiva y equitativa de calidad y promover oportunidades de aprendizaje permanente para todos" (ONU, 2015, p. 1). Se insta hacia el 2030 la meta de asegurar el acceso igualitario a la educación. Argentina adhiere a estos objetivos tomándolos como una política más de Estado y de Derechos Humanos. 
En nuestro país, en el año 2006, se sanciona la Ley de Educación Nacional -LEN26.206 (tercera en la historia argentina), en la cual se establece que la educación y el conocimiento son un bien público y un derecho personal y social cuyo acceso, permanencia y egreso deben ser garantizados por el Estado y sus instituciones (art. 2). El Estado y sus ministerios son los responsables de garantizar una educación integral, permanente y de calidad, enmarcada en la igualdad, gratuidad y equidad para todas las personas en los niveles y modalidades del Sistema Educativo (art. 4, art. 42).

En la Ley de Educación Nacional que estructura el Sistema Educativo Argentino, se afirma que la Educación Especial se rige por el principio de inclusión, sin embargo, al mismo tiempo es definida como aquella destinada a asegurar el derecho a la educación de las personas con discapacidades, brindando atención educativa en aquellas trayectorias que no puedan ser abordadas por la educación común (art. 42), resultando así poco consistente en su posicionamiento desde un marco inclusivo. De hecho, usa de modo alternado los términos de integración con inclusión, sin destacar sus diferencias. Cabe aclarar que esta ley es un poco anterior a la CDPD (ONU, 2006).

En el año 2016, el Consejo Federal de Educación emite una resolución de cara a satisfacer los derechos incumplidos de las personas con discapacidad en el ámbito educativo. Dicha resolución -n. ${ }^{\circ}$ 311/16- cobra denominación bajo el nombre Promoción, acreditación, certificación y titulación de estudiantes con discapacidad. Esta resolución insta a profundizar en la cultura inclusiva al interior de los establecimientos educativos. Para ello, promueve la implementación de ajustes razonables, apoyos necesarios con abordaje institucional, accesibilidad, corresponsabilidad entre niveles y modalidades, e identificación de barreras en la participación y aprendizaje para el acompañamiento de las trayectorias escolares. Sin embargo, manifiesta algunos puntos en contradicción con la misma Convención antes citada. Por ejemplo, plantea la elaboración de un Proyecto Pedagógico Individual para la Inclusión -PPI- para todos/as los/as estudiantes con discapacidad que asisten a escuelas comunes/regulares, el cual define tanto las propuestas de enseñanza como de evaluación y certificación individuales; además, continúa responsabilizando a la educación especial la intervención en los procesos educativos cuando se trata de un/a estudiante con discapacidad. Esto denota una postura segregadora que se contrapone al enfoque inclusivo y del diseño universal propuesto en la Convención.

En la actualidad, los/as profesionales de apoyo que orientan y acompañan las trayectorias educativas de estudiantes con discapacidad incluidos en escuelas comunes/ regulares pueden provenir de la Educación Especial o de profesionales independientes subvencionados por el área de salud del Gobierno argentino. Por un lado, el agente educativo que pertenece a la modalidad de Educación Especial tiene como función trabajar en corresponsabilidad con los/as docentes a cargo del estudiante con discapacidad, para el diseño y la implementación de ayudas y estrategias de enseñanza y de aprendizaje que garanticen una educación de calidad para todos/as los/as estudiantes, tal como enuncian los marcos normativos mencionados. Cabe aclarar que está en un proceso de transición respecto a su refuncionalización como centro de recursos especializados de apoyo a la inclusión en el ámbito de la educación pública/privada común. Sin embargo, y desde nuestra mirada, las políticas de Estado al respecto no 
ATENCIÓN TEMPRANA EN TIEMPOS DE COVID-I9: INVESTIGAR LA/S REALIDAD/ES DE LA TELEINTERVENCIÓN EN LAS PRÁCTICAS CENTRADAS EN LA FAMILIA M. E. YADAROLA, C. DEL VALLE ERRAMOUSPE, F. TOMÁS GONZÁLEZ...

son suficientes, coherentes ni eficientes para la plena realización. Esto se traduce en la creación de nuevas escuelas especiales, en el fomento de estas con enfoques segregacionistas, en la falta de un posicionamiento ideológico compartido respecto a la discapacidad y a la inclusión, entre otras cuestiones, que dificultan llevar adelante una planificación prospectiva y con metas claras hacia una profunda transformación del sistema educativo para que sea inclusivo.

Por otro lado, como decíamos, los/las profesionales de apoyo particulares que acompañan en forma individual las trayectorias educativas de estudiantes con discapacidad en procesos de inclusión dependen del sistema de salud nacional, aspecto que obstruye la posibilidad de un trabajo enfocado en el contexto escolar e institucional. La familia de cada estudiante es quien contrata al profesional. Esta situación ocasiona el solapamiento entre los distintos abordajes y la falta de unificación en criterios que sean realmente inclusivos.

Es la responsabilidad de las políticas públicas el garantizar la inclusión, la igualdad y la equidad, propiciando las condiciones para que todos/as puedan aprender con calidad. El Estado debe asegurar que todos/as tengan igualdad de oportunidades y condiciones para participar y aprender, debe ofrecer propuestas equitativas e inclusivas de formación en el marco de una escuela y aula común, brindando los accesos, los apoyos y los ajustes que cada estudiante requiere. A su vez, cada institución educativa tiene la responsabilidad de planificar y llevar adelante las acciones hacia el logro de los objetivos establecidos, traduciendo los grandes desafíos de las políticas educativas en objetivos institucionales, generando las mejores estrategias para transitar este camino.

Remarcamos que la inclusión educativa de las personas con discapacidad es un derecho. Siguiendo a Echeita y Ainscow (2010), la inclusión educativa como proceso refiere a la presencia, participación y aprendizaje de todos/as los/as estudiantes en la escuela y en el aula común/regular, sin excepciones. Requiere eliminar y/o minimizar barreras (por ejemplo, físicas, actitudinales, comunicativas, curriculares) que aún se sostienen desde un paradigma homogeneizador.

Si las personas con discapacidad son quienes deben adaptarse a los requisitos de las instituciones comunes, este proceso marca una integración, no una inclusión. En la inclusión son la escuela y el aula los que deben estructurarse y transformarse para enseñar a cada estudiante, sin discriminación. Además, cabe agregar que "la integración no garantiza automáticamente la transición de la segregación a la inclusión” (ONU, 2016, p. 4).

Una enseñanza a través de agrupamientos variados y flexibles de trabajo en el aula diversa, priorizando el trabajo colaborativo entre estudiantes con y sin discapacidad, favorece el progreso del aprendizaje de todos/as y el desarrollo de valores humanos necesarios para fortalecer una sociedad más inclusiva. Para ello, es necesario que las prácticas de enseñanza se vean transformadas hacia un Diseño Universal de Enseñanza -DUE- que implica la "estructuración de la enseñanza para el aprendizaje de todos, desde un enfoque contextual y holístico. Aplicar el DUE en el aula común inclusiva beneficia a todos los estudiantes, con o sin discapacidad, porque está diseñado para todos y cada uno" (Yadarola, 2016a, p. 27). Esta es una tarea que debe ser colegiada y colaborativa, entre docentes de la escuela y los/las profesionales de apoyo, 
para un trabajo escolar situacional y contextual, que tenga en cuenta la singularidad de cada estudiante como de cada espacio escolar e institucional en su complejidad.

El rol docente es fundamental, de manera que la formación de cada estudiante oriente a desarrollar aprendizajes relevantes, su autodeterminación y mejora de su calidad de vida. Los/as estudiantes con discapacidad intelectual presentan formas y modos de aprender singulares, pero, fundamentalmente, necesitan de un docente que los/las aliente y guíe para desarrollar al máximo sus capacidades; necesitan de redes naturales de apoyo entre compañeros/as con y sin discapacidad, como mediadores en el aprendizaje. Este marco de la inclusión educativa debe hoy también verse reflejado en la tarea de enseñanza remota de emergencia.

Retomando el espíritu del objetivo n. ${ }^{\circ} 4$ de los ODS, los conceptos de equidad e inclusión educativa son esenciales para alcanzar una atención de calidad, respetando los ajustes razonables y apoyos necesarios de los/as estudiantes. La importancia que cobran las prácticas educativas, visibilizadas en una planificación flexible y con diversidad de opciones, pone de manifiesto que todos los estilos de aprendizaje están presentes, son valorados y tienen lugar en la propuesta docente. La relación del/la docente como mediador/a y sostén emocional que acompañe el proceso de aprendizaje es vital en el acto pedagógico. Y cobra mayor relevancia cuando esas ayudas llegan a todos/as, incluido el/la estudiante con discapacidad intelectual, más en las circunstancias actuales de gran complejidad e incertidumbre.

En el contexto de la institución educativa y del oficio de enseñar, el profesor o la profesora tienen diversas maneras de relacionarse con sus estudiantes, y ese modo de relacionarse y de “querer" incidirá en la formación de sus estudiantes para que alcancen su plena realización personal. Abramowski (2012) afirma que la relación educativa implica un encuentro entre desconocidos, en sentido estricto, y que "un afecto docente que es propio del querer enseñar unos contenidos a un conjunto de alumnos" es aquel afecto docente que "se interesa y persevera para que algo del orden de las enseñanzas pueda tener lugar” (Abramowski, 2012, p. 13). Podemos agregar que es aquel docente que dispone del tiempo para comunicarse y escuchar a sus estudiantes, que desarrolla una relación pedagógica desde la empatía y la comprensión mutua, que reaprende en colaboración su tarea como docente, que planifica y pone en funcionamiento una enseñanza remota de emergencia de calidad, para todos/as y cada uno/a de sus estudiantes.

\section{Método}

El objetivo del estudio fue indagar si las prácticas de enseñanza desarrolladas en tiempos de aislamiento social preventivo obligatorio en Argentina favorecen la inclusión, los aprendizajes y la autonomía de los/as estudiantes con discapacidad intelectual, en opinión de sus profesionales de apoyo a la inclusión y de sus familias.

Se partió desde el siguiente problema de investigación: ¿las prácticas de enseñanza desarrolladas en este período de aislamiento social preventivo obligatorio en Argen- 
ATENCIÓN TEMPRANA EN TIEMPOS DE COVID-I 9: INVESTIGAR LA/S REALIDAD/ES DE LA TELEINTERVENCIÓN EN LAS PRÁCTICAS CENTRADAS EN LA FAMILIA M. E. YADAROLA, C. DEL VALLE ERRAMOUSPE, F. TOMÁs GONZÁLEZ...

tina favorecen la inclusión, los aprendizajes y la autonomía de los/as estudiantes con discapacidad intelectual?

Se realizó este trabajo con un diseño exploratorio con énfasis en lo descriptivo, ya que se trata de una situación no indagada anteriormente, en el marco de un contexto inédito.

Dada la dificultad de acceder a las escuelas, por estar aún cerradas en nuestro país (salvo excepciones), se procedió a seleccionar la muestra en función de la viabilidad de acercarnos a la realidad de los/as estudiantes con discapacidad intelectual en procesos de inclusión. El equipo de investigación cuenta con una mayor proximidad con profesionales de apoyo. Ello motivó a seleccionar como unidades de observación a profesionales de apoyo a los procesos de inclusión de estudiantes con discapacidad intelectual incluidos en escuelas comunes/regulares y las familias de dichos estudiantes.

El muestreo es de tipo intencional. Se contactó a diversos profesionales y familias a través de diversos canales (email, WhatsApp, llamadas telefónicas, etc.) durante las primeras semanas de septiembre de 2020. Respondieron al cuestionario 75 profesionales de apoyo y 40 familias, de modo anónimo, por lo cual no puede inferirse que refieran a los mismos estudiantes con discapacidad intelectual. Entonces, dichos profesionales describieron la situación de 75 estudiantes y las familias, de otros 40.

Se utilizó para ello un cuestionario elaborado por el equipo de investigación, teniendo como base una guía de observación antes armada e implementada para indagar la situación de inclusión de estudiantes con discapacidad en las escuelas y en las aulas comunes.

El cuestionario quedó conformado, en general, con preguntas cerradas, para facilitar el procesamiento de datos, y algunas preguntas abiertas. Dicho cuestionario se aplicó, a través de un formulario del Google Drive, a profesionales de apoyo y a familias de estudiantes con discapacidad intelectual, incluidos en escuelas comunes.

El análisis de los datos se realizó desde un enfoque cuantitativo, con algunos elementos cualitativos que surgieron de los comentarios de los encuestados y de la interpretación de los datos. Se elaboraron algunas figuras con las frecuencias de las respuestas consideradas más relevantes, posibilitando la comparabilidad entre las opiniones de profesionales de apoyo y las familias.

\section{Principales resultados}

Para este estudio, por un lado, recabamos información sobre los procesos de inclusión educativa de 75 estudiantes con discapacidad intelectual que asisten a instituciones educativas comunes, a través de las respuestas de sus docentes de apoyo. Estos estudiantes residen en su mayoría en diferentes localidades de la Provincia de Córdoba (68 \%), mientras que el resto lo hace en Córdoba Capital (22,7 \%) y en localidades de otras provincias (9,3\%). Las instituciones educativas a las que asisten corresponden a gestión estatal (70,7 \%) y gestión privada (29,3\%). Los/las profesionales de apoyo encuestados se encuentran acompañando procesos de inclusión en el nivel secundario $(66,7 \%)$ y en el nivel primario $(33,3 \%)$. Por otra parte, recibimos información de 40 familias que tienen un miembro con discapacidad intelectual en 
edad escolar que asiste a escuela común. Estas familias residen en su mayoría en el interior de la Provincia de Córdoba (67,5\%), mientras que el resto de los encuestados residen en localidades de otras provincias (20 \%) y en Córdoba Capital $(12,5 \%)$. El tipo de gestión de la escuela a la que asisten estos estudiantes es estatal (55\%) y privada (45\%). La mitad se encuentran cursando el nivel primario (50 \%), un importante porcentaje corresponde al nivel secundario (42,5\%), siendo la menor cantidad quienes asisten a los niveles inicial y superior $(7,5 \%)$.

En cuanto a los recursos tecnológicos de los que disponen los/as estudiantes para el aprendizaje desde la virtualidad, según sus profesionales de apoyo, encontramos que la mayoría comparte con algún miembro de su familia la computadora $(38,7 \%$ ) y el celular (56 \%). El acceso a internet por banda ancha alcanza mayor porcentaje $(60 \%)$ mientras que el resto tiene datos reducidos (14,7\%).

Según las familias, los recursos tecnológicos de que disponen para acompañar el aprendizaje desde la virtualidad, en mayores porcentajes, son computadora comparti$\mathrm{da}(47,5 \%)$ y celular compartido (45\%) con otros miembros de la familia. El acceso a internet de banda ancha es el utilizado por la mayor parte de las familias $(70 \%)$ en tanto el resto utiliza datos disponibles en el celular $(7,5 \%)$ o no tiene acceso $(2,5 \%)$.

Tanto los/las profesionales de apoyo como las familias expresaron que en sus hogares los/as estudiantes con discapacidad reciben el apoyo, principalmente, de la madre para resolver las tareas escolares ( $89,3 \%$ y $85 \%$ respectivamente).

Figura 1. Destinatarios de la planificación del/de la docente de la escuela según profesionales de apoyo

Destinatarios de la planificacion del/la docente de la escuela

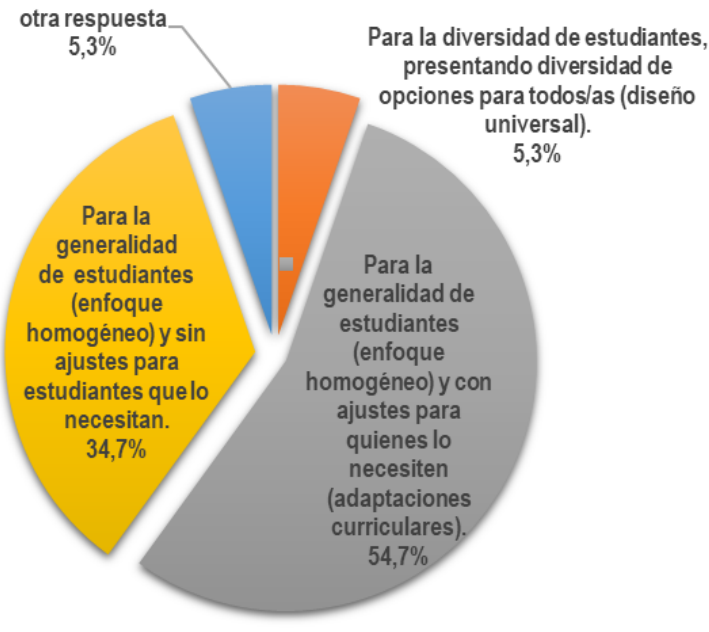

Ediciones Universidad de Salamanca / CC BY-NC-ND

Siglo Cero, vol. 52, número extraordinario, 2021, pp. 163-182 
Se buscó indagar en la voz de los/as profesionales de apoyo si los/as docentes de escuela planifican el desarrollo de sus clases para todos/as sus estudiantes por igual, desde un enfoque homogéneo, posibilitando o no que el/la estudiante con discapacidad pueda acceder a los ajustes y apoyos que requiere o si el enfoque de su planificación, así como de las estrategias de enseñanza, ofrece variedad de opciones, a modo del diseño universal.

En la Figura 1 se observa que la mayoría de los/as profesionales de apoyo (54,7 \%) consideran que los/las docentes planifican desde un enfoque homogéneo para la generalidad de sus estudiantes y realizando adaptaciones curriculares para los/as estudiantes que lo necesitan, dentro de los que se encuentra el/la estudiante con discapacidad intelectual. Solo un 5,3\% considera que los/las docentes planifican para la diversidad de sus estudiantes, ofreciendo variedad de opciones con una mirada desde el diseño universal. Es de resaltar que, según los/as profesionales de apoyo, en un importante porcentaje $(34,7 \%)$, los/las docentes planifican de modo homogéneo y sin ajustes para los/as estudiantes que lo requieren, dejando así a sus estudiantes con discapacidad intelectual sin apoyos y dificultando el acceso para poder aprender y participar en igualdad de condiciones que sus compañeros.

FiguRA 2. Planteo de estrategias de enseñanza que responden a las necesidades de la diversidad de estudiantes, según profesionales de apoyo

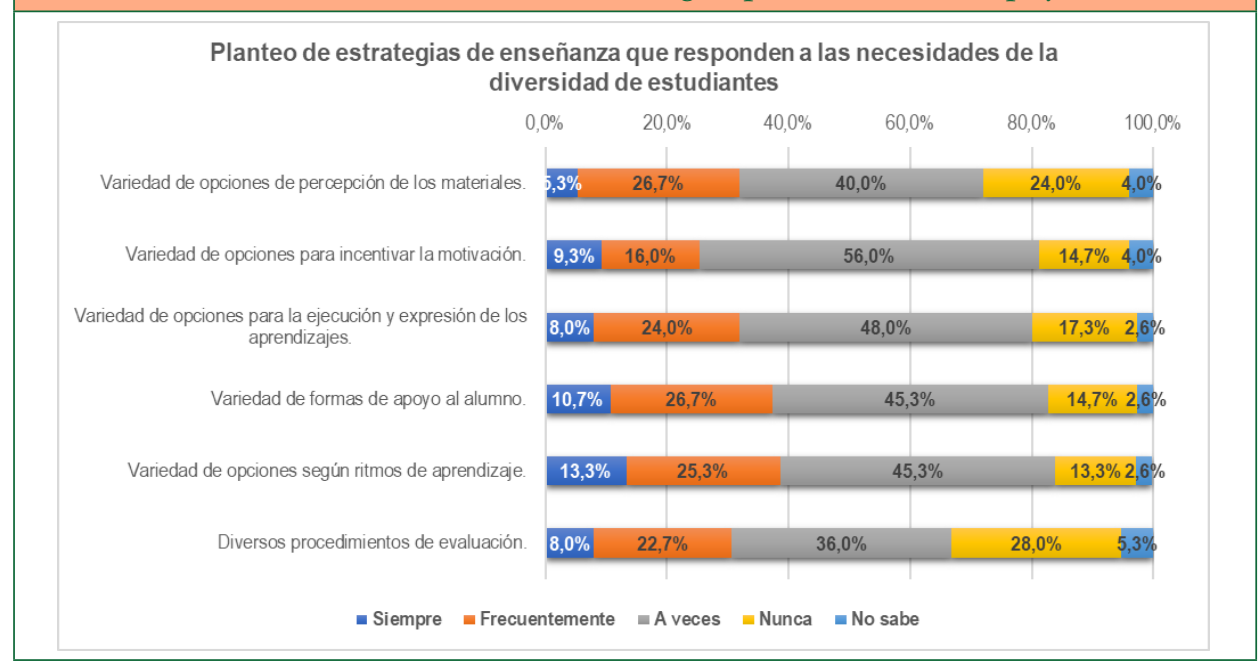

La Figura 2 resulta consistente con el anterior análisis. Así, los más altos porcentajes de profesionales de apoyo (entre $56 \%$ y un $36 \%$ ) indican que los/las docentes solo $A$ veces ofrecen a sus estudiantes variedad de opciones (de percepción de los materiales, para incentivar la motivación, para la ejecución y expresión de los aprendizajes, según ritmos de aprendizaje), variedad de formas de apoyo al/a la estudiante y diversos procedimientos de evaluación. En este último indicador es donde un $28 \%$ 
de encuestados/as afirma que Nunca se realiza el planteo de diversidad de procedimientos de evaluación. Además, un 24 \% considera que Nunca el/la docente ofrece variedad de opciones de percepción de los materiales.

Ahora bien, si en general los/as docentes están planificando desde un enfoque homogéneo y realizando ajustes (o no) para el/la estudiante con discapacidad, pero solo $A$ veces o Nunca ofrecen a la clase variedad de opciones desde un diseño universal, difícilmente podemos dar cuenta de un aula inclusiva, donde se enseña a la diversidad según ritmos y modos de aprender. Más particularmente, queda obstaculizada una evaluación que tenga en cuenta las necesidades de ajustes y acceso de cada estudiante, lo que muy puntualmente se convierte en una barrera a las posibilidades de un/a estudiante con discapacidad intelectual de acreditar los aprendizajes logrados y progresar en su educación.

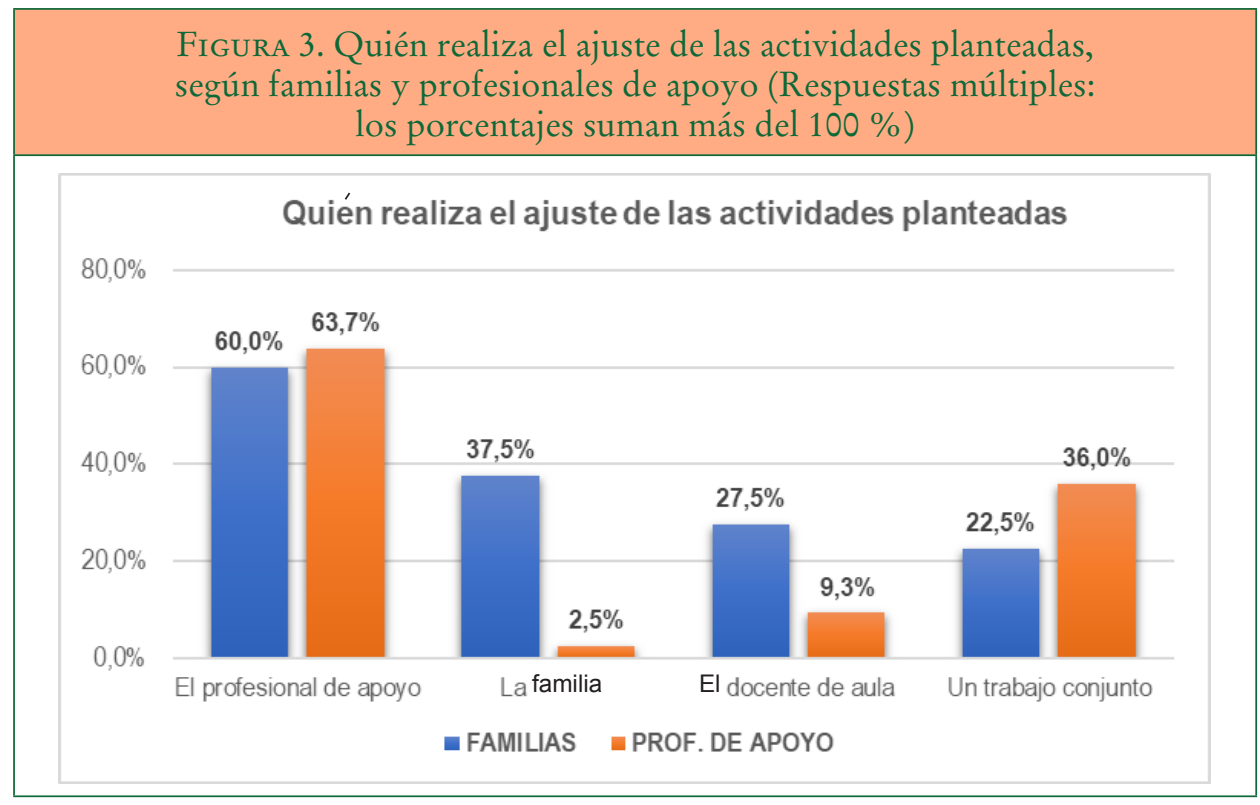

Interesó identificar en este año de aislamiento preventivo en Argentina quién o quiénes están realizando los ajustes de las actividades planteadas en la escuela, para analizar si se trata de una tarea compartida o que recae en un solo actor educativo. Cabe aclarar que los encuestados podían indicar más de una respuesta (ver Figura 3), por lo que la suma de porcentajes es mayor al $100 \%$.

Tanto familias como profesionales de apoyo en mayor porcentaje indican que estos ajustes los realiza el/la profesional de apoyo (60 \% y 63,7 \%, respectivamente). En segundo lugar, mientras un 37,5\% de familias afirma que están realizando los ajustes la propia familia, un $36 \%$ de profesionales de apoyo indican que se trata de un trabajo en conjunto (entre docentes y profesionales de apoyo o entre profesionales de apoyo 
y familias o entre estos tres actores institucionales). Cabe destacar que son menos familias y menos aún profesionales de apoyo quienes afirman que dichos ajustes los realiza el docente de aula ( $27,5 \%$ y $9,3 \%$, respectivamente). Por tanto, pareciera que, en la voz de los encuestados, la responsabilidad de realizar ajustes curriculares, apoyos y accesos recae en el/la profesional de apoyo, resultando un dato preocupante. Es de considerar que en general los/las profesionales de apoyo son externos a la escuela, lo que podría estar indicando la falta de compromiso de las instituciones educativas con la inclusión, en general, y con el/la estudiante con discapacidad intelectual, en particular. Asimismo, interpela sobre la función docente y su compromiso con la enseñanza para la diversidad de sus estudiantes.

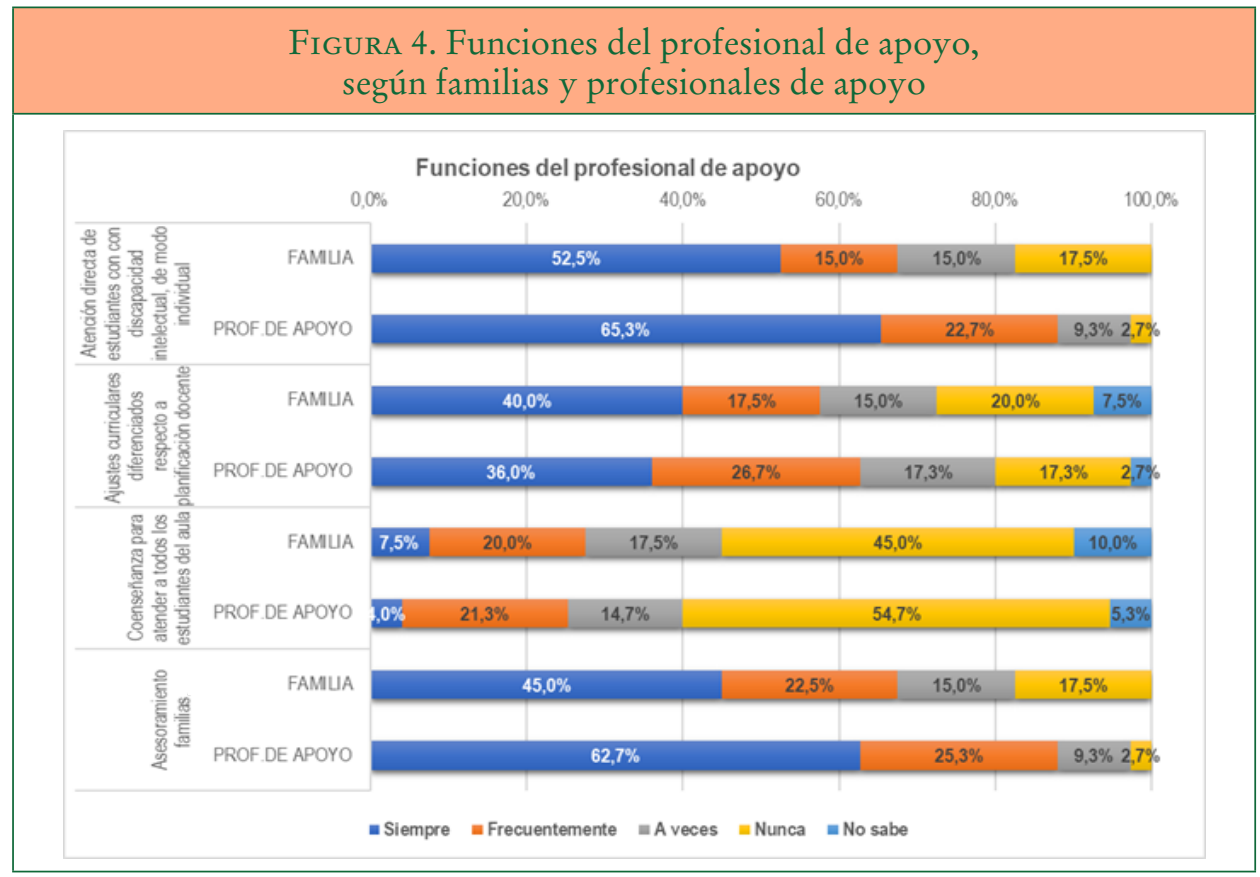

Coherentemente con lo examinado en el punto anterior, al analizar las funciones del profesional de apoyo según familias y profesionales de apoyo (Figura 4) en más altos porcentajes indican, por un lado, que Siempre las tareas asumidas son Atención directa de estudiantes con discapacidad intelectual, de modo individual (52,5\% y 65,3\%, respectivamente); Asesoramiento familias (45\% y 62,7 \%, respectivamente), y Ajustes curriculares diferenciados respecto a la planificación docente (40\% y $36 \%$, respectivamente).

Sin embargo, por otro lado, familias y profesionales de apoyo señalan que Nunca asumen como función la Coenseñanza para atender a todos los/as estudiantes del aula (45\% y 54,7 \%, respectivamente). Entonces, las funciones de profesionales de apoyo 
refieren al estudiante con discapacidad intelectual en particular, pero descontextualizándolo del espacio escolar y de las actividades de la clase, reforzando la barrera para el desarrollo de un aula inclusiva que enseñe a la diversidad de estudiantes, con o sin discapacidad, desde un trabajo colaborativo de coenseñanza. Las políticas públicas argentinas, antes analizadas, enfocadas más hacia procesos de integración e incluso de segregación, más que de inclusión, probablemente estén incidiendo en este sentido.

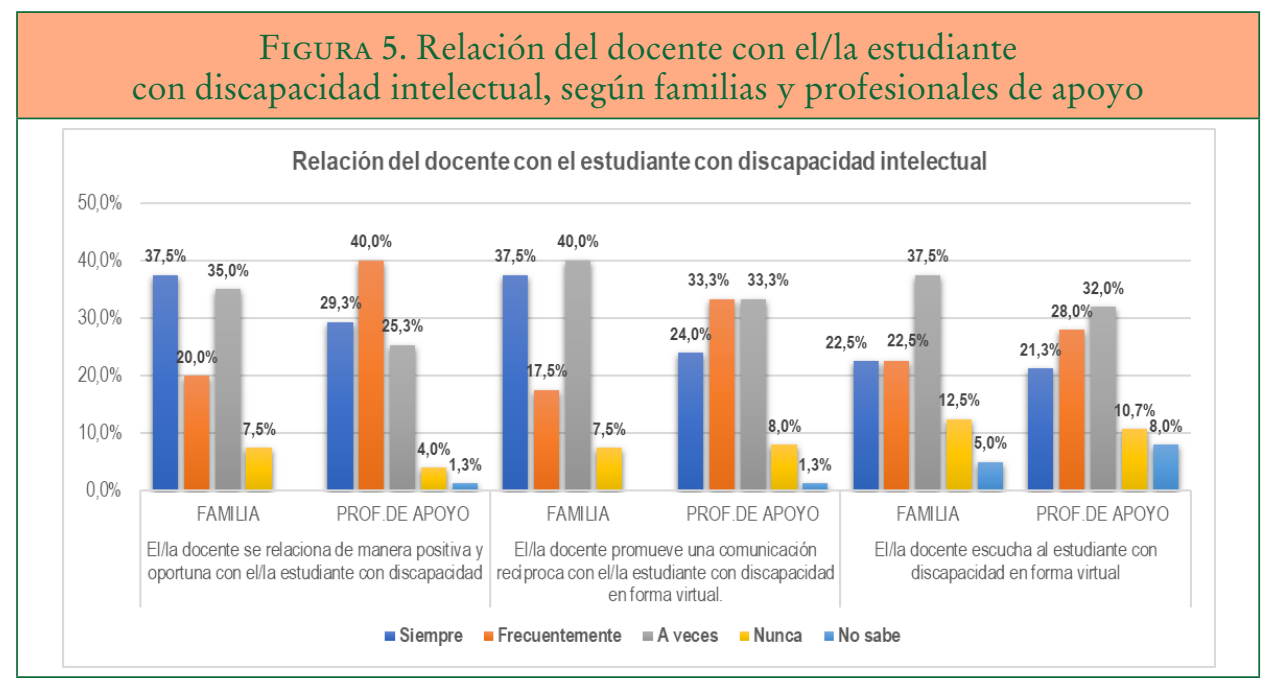

Otro aspecto que se consultó fue respecto a la relación del/de la docente de aula con el/la estudiante con discapacidad intelectual (Figura 5) en estos tiempos de aislamiento social preventivo obligatorio en Argentina y de enseñanza remota de urgencia.

Si sumamos las categorías Siempre y Frecuentemente podemos observar que la mayoría, tanto familias como profesionales de apoyo, consideran que el/la docente se relaciona de manera positiva y oportuna con el/la estudiante con discapacidad (57,5\% y 69,3\%, respectivamente) y que el/la docente promueve una comunicación recíproca con el/la estudiante con discapacidad en forma virtual (55\% y 57,3\%, respectivamente).

Ahora bien, es alto el porcentaje de familias y profesionales de apoyo que consideran que solo es $A$ veces (entre un $40 \%$ y un $25,3 \%$ ) y menos marcaron Nunca (entre un $8 \%$ y un $4 \%$ ) que el/la docente se relaciona positivamente promoviendo una comunicación recíproca. Estos datos dan cuenta de que un importante número de estudiantes con discapacidad intelectual hoy tienen una escasa comunicación y relación con sus docentes.

En cuanto al indicador el/la docente escuch a al estudiante con discapacidad en forma virtual, las respuestas resultan relativamente más parejas. Las familias y profesionales de apoyo indican que la escucha docente es Siempre/Frecuentemente (45 \% y 49,3 $\%$, respectivamente) o $A$ veces/Nunca (50 \% y $42,7 \%$, respectivamente). Entonces, 


\section{ATENCIÓN TEMPRANA EN TIEMPOS DE COVID-I9: INVESTIGAR LA/S REALIDAD/ES DE LA TELEINTERVENCIÓN EN LAS PRÁCTICAS CENTRADAS EN LA FAMILIA M. E. YADAROLA, C. DEL VALLE ERRAMOUSPE, F. TOMÁS GONZÁLEZ...}

se aumenta aquí el porcentaje de encuestados que señalan una menor frecuencia en la escucha al estudiante con discapacidad en estas instancias que estamos viviendo de un formato remoto de enseñanza, en un contexto complejo y de incertidumbre general.

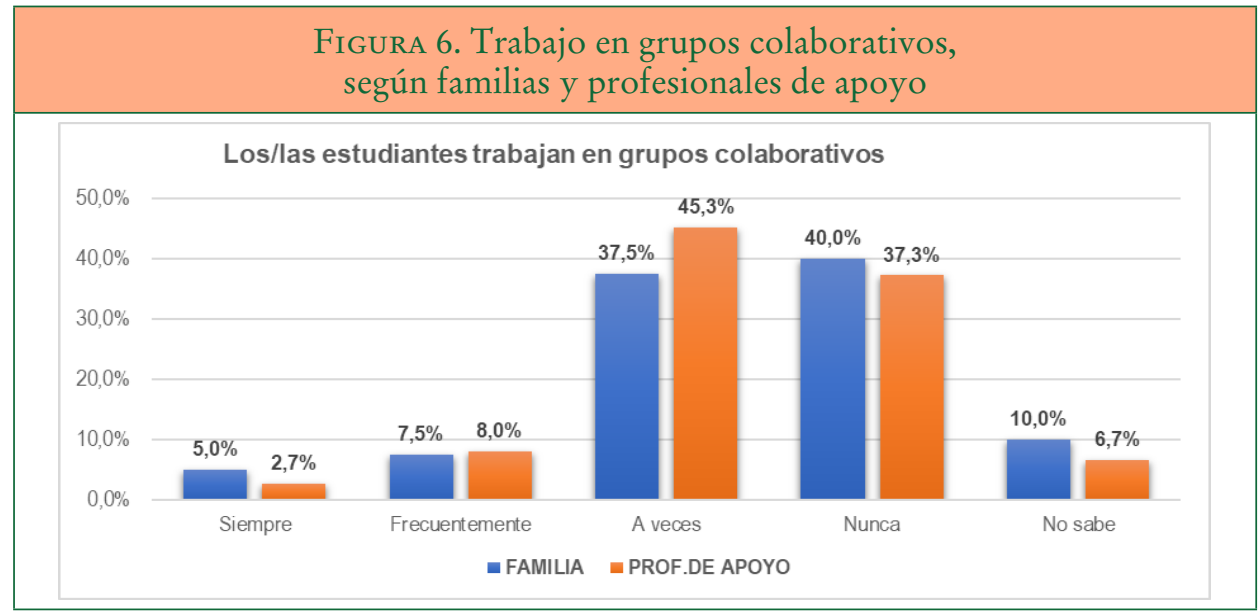

Además de la menor frecuencia de una positiva relación entre docente con el/la estudiante con discapacidad, se agregan las escasas estrategias implementadas para el trabajo colaborativo entre estudiantes (ver Figura 6). De esta manera, las familias y profesionales de apoyo revelan que en general es $A$ veces $(37,5 \%$ y $45,3 \%$, respectivamente) o Nunca (40\% y $37,3 \%$, respectivamente) que los/as estudiantes trabajan en grupos colaborativos. Es importante recordar que el aprendizaje se desarrolla en colaboración y que estas instancias de trabajo grupal promueven la zona de desarrollo próximo, en términos vygostkianos, además de ser una estrategia favorecedora de redes de compañerismo y de desarrollo de habilidades interpersonales. Además, hay que tener en cuenta que hay muchos modos virtuales de trabajar en grupo, sea con la utilización de, por ejemplo, grupos de WhatsApp, Facebook, documentos compartidos, plataformas con sesiones grupales, etc., que cada docente puede elegir en función de la disponibilidad de recursos de sus estudiantes. Es así como la falta de una utilización y fomento de estrategias cooperativas de modo frecuente se constituye en una barrera para el aprendizaje, en especial para aquellos estudiantes con discapacidad intelectual que requieren de mayores apoyos y mediaciones en sus aprendizajes, así como el fortalecimiento de redes naturales de apoyo entre compañeros. 


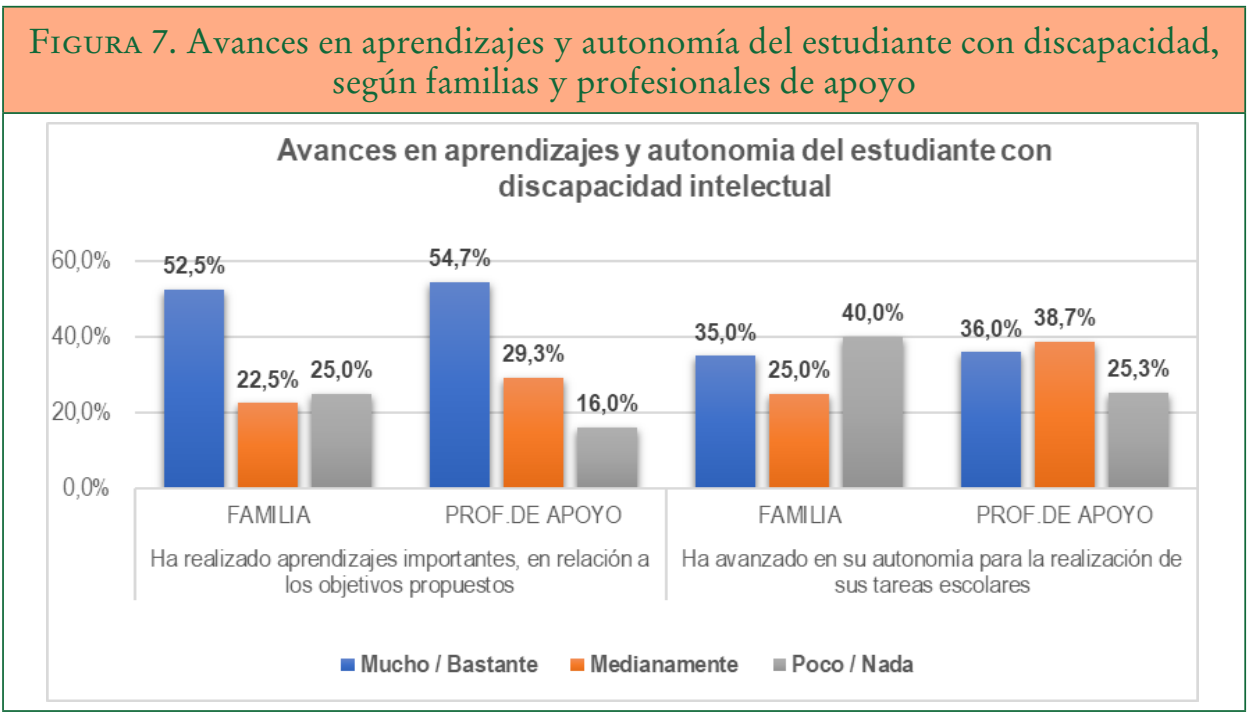

Uno de los aspectos más relevantes que se preguntó a las familias y los/las profesionales de apoyo fue si los/as estudiantes habían logrado aprendizajes importantes en relación con los objetivos propuestos (ver Figura 7). Aquí observamos que el 52,5\% de las familias respondió que sus hijos aprendieron Mucho o Bastante; el 22,5\% opina que sus hijos con discapacidad intelectual han aprendido Medianamente, y el $25 \%$ considera que han aprendido Poco o Nada. Cabe nuevamente acentuar que los encuestados responden cada uno/a por un/a estudiante con discapacidad intelectual, por tanto, son porcentajes, asimismo, de estudiantes con discapacidad que dan cuenta de sus aprendizajes y su autonomía o de sus dificultades en ello.

Estos porcentajes son muy elevados en relación con el total de encuestados, ya que, si sumamos las respuestas que incluyen medianamente, poco y nada, tenemos como resultado que el 47,5\% de las familias observó que no se han producido aprendizajes suficientes, lo cual nos arroja la hipótesis de que la enseñanza remota de emergencia estaría aumentando aún más las barreras para el aprendizaje y la participación de los/as estudiantes con discapacidad intelectual. Asimismo, puede decirse que las propuestas pedagógicas didácticas impartidas y/o el acompañamiento pedagógico de las familias no estarían generando los resultados esperados en función de los objetivos. Ello también obstaculizado por las dificultades en el acceso a la tecnología necesaria en los tiempos de uso requeridos.

La mayoría de los/las profesionales de apoyo (54,7 \%) consideran que los/as estudiantes han logrado aprender Mucho o Bastante. Un 29,3\% opinan que han aprendido Medianamente y el $16 \%$ de los/las profesionales de apoyo consideran que se ha aprendido Poco y Nada.

El porcentaje más alto en el logro de aprendizaje según la opinión de los/las profesionales de apoyo podría deberse o estar directamente implicado con su rol en tiempos 
ATENCIÓN TEMPRANA EN TIEMPOS DE COVID-I9: INVESTIGAR LA/S REALIDAD/ES DE LA TELEINTERVENCIÓN EN LAS PRÁCTICAS CENTRADAS EN LA FAMILIA M. E. YADAROLA, C. DEL VALLE ERRAMOUSPE, F. TOMÁS GONZÁLEZ...

de pandemia, donde la mayoría de los mismos han quedado a cargo de la preparación, planificación y adecuación de objetivos de aprendizaje para los/as estudiantes con discapacidad intelectual (según lo ya analizado en las Figuras 3 y 4). Este contacto estrecho con la familia del/de la estudiante y los contenidos estaría relacionado con la posibilidad de observación directa de resultados sobre los objetivos de aprendizaje y, por ende, las respuestas tienden a ser positivas y arrojan un porcentaje mayor.

No obstante, si tomamos nuevamente los resultados de Medianamente y Poco o Nada, en la opinión de los/las profesionales de apoyo el 45,3 \% de los mismos consideran que no se han producido aprendizajes suficientes en relación con los objetivos.

Tanto en la opinión de las familias como de los/las profesionales observamos cifras altas y alarmantes que nos muestran el aumento de las barreras y las desigualdades.

En la Figura 7 asimismo se focalizó en identificar si los/as estudiantes avanzaron en autonomía en la realización de las tareas escolares. Un $35 \%$ de las familias opinan que han logrado Mucho o Bastante, un $25 \%$ Medianamente y un $40 \%$ Poco y Nada. Esta última cifra resulta alarmante y preocupante, dado que una de las habilidades más importantes a desarrollar en las personas con discapacidad intelectual es la autonomía. Asimismo, podría inferirse que, al haber logrado poca autonomía en la realización de las tareas, entonces la asistencia y el apoyo por parte de las familias podrían haberse incrementando, generando situaciones de dependencia de los/as estudiantes.

En la opinión de los/las profesionales de apoyo, las cifras cambian en relación con lo arrojado por las familias. Así observamos que un $36 \%$ opina que han avanzado en autonomía Mucho o Bastante; un 38,7 \% Medianamente, y un 25,3\% Poco y Nada. Cabe aquí recordar que la mayoría de los/as profesionales de apoyo refieren a estudiantes con discapacidad que asisten al nivel secundario (66,7\%), en cambio, las familias refieren en su mayoría a estudiantes en el nivel primario (50\%). Esto puede influir en la mayor autonomía que observan los/las profesionales de apoyo por tratarse de estudiantes de mayor edad.

\section{Conclusiones}

Iniciamos este trabajo de investigación preguntándonos si las prácticas educativas desarrolladas en tiempos de aislamiento social preventivo obligatorio en Argentina favorecen la inclusión, los aprendizajes y la autonomía de los/as estudiantes con discapacidad intelectual.

En estos momentos de expansión de la pandemia de COVID-19, el Estado argentino no ha podido garantizar el acceso de sus estudiantes a la enseñanza remota de emergencia en igualdad de condiciones. La tecnología necesaria y la conectividad a internet de los/as estudiantes quedó a cargo de las familias, quienes en la mayoría de los casos debieron compartir dispositivos tecnológicos. Esto obstaculiza la participación en las clases y actividades virtuales a muchos/as estudiantes con discapacidad intelectual y, con ello, su oportunidad de contar con un encuentro pedagógico necesario con sus docentes. 
De acuerdo con lo observado, la inclusión educativa de los/as estudiantes con discapacidad depende de las acciones de cada institución educativa o cada docente, quedando expuestos a procesos de integración en la mayoría de los casos. Solo una minoría accede a una educación más inclusiva, según lo manifestado por los encuestados. En relación con la tarea docente observamos que, en general, en sus planificaciones prevalece el enfoque homogéneo, sin ofrecer ajustes curriculares o quedando a criterio del profesional de apoyo las adaptaciones curriculares, la atención directa con el/ la estudiante con discapacidad intelectual y el asesoramiento a las familias. Son pocos los docentes que trabajan en colaboración, desde la coenseñanza con profesionales de apoyo y ofrecen a sus estudiantes variadas estrategias y opciones desde un enfoque del diseño universal.

En cuanto al vínculo del/de la docente con el/la estudiante con discapacidad intelectual, se observa en general una buena relación y una comunicación recíproca, pero muchos de los estudiantes en un alto porcentaje tienen un escasa comunicación y relación, llegando a ser nula en algunos casos, especialmente en la escucha hacia estos estudiantes. A esto se le suma el escaso trabajo en grupos cooperativos desarrollados desde la virtualidad, como estrategia incorporada a la enseñanza remota de emergencia, por ser una estrategia que promueve el desarrollo integral y los aprendizajes de cada estudiante, fortaleciendo la inclusión. Es de recordar que el proceso de enseñanza es, antes que nada, un encuentro humano, que necesita del diálogo y de un vínculo desde el afecto y la empatía.

Para las familias y los/las profesionales de apoyo, un porcentaje relevante de estudiantes con discapacidad intelectual no han alcanzado los objetivos de aprendizaje propuestos, ni la autonomía esperada. Es de retomar, además, que muchos no son escuchados por sus docentes, aspecto que acentúa su situación de vulnerabilidad, de invisibilidad.

Las barreras al aprendizaje y la participación con las que se encuentran los/as estudiantes con discapacidad intelectual se sustentan en las políticas educativas argentinas y se plasman en prácticas educativas homogeneizadoras y segregadoras.

Apelamos a que el Estado argentino realice un cambio de mirada para revertir la situación en la que se encuentran los/las estudiantes con discapacidad intelectual, haciendo valer el modelo social de la discapacidad y su enfoque de educación inclusiva como un derecho irrenunciable e inapelable de los/as estudiantes con y $\sin$ discapacidad, poniendo en contexto todos los accesos, apoyos y ajustes necesarios que garanticen una mejor calidad de vida de todas aquellas personas en condiciones de vulnerabilidad. Yadarola (2016b) afirma que la inclusión debe ser encarada como parte de un proyecto de vida de una persona con discapacidad, como parte de un proyecto de sociedad:

La inclusión de una persona con discapacidad intelectual en todos los ámbitos de la vida hace que esa persona pueda tener una vida más plena, participando como cualquier otro miembro y ciudadano de la sociedad. Ese niño incluido en la escuela se pueda proyectar como adulto incluido a lo largo de su vida y en todos los ámbitos, porque es uno más en la diversidad (Yadarola, 2016b, p. 8). 
ATENCIÓN TEMPRANA EN TIEMPOS DE COVID-I 9: INVESTIGAR LA/S REALIDAD/ES DE LA TELEINTERVENCIÓN EN LAS PRÁCTICAS CENTRADAS EN LA FAMILIA M. E. YADAROLA, C. DEL VALLE ERRAMOUSPE, F. TOMÁS GONZÁlEZ...

Para ello, será relevante analizar las condiciones necesarias para el aprender contemplando la diversidad de formas, de situaciones, de recursos, de apoyos, ajustes y accesibilidad, así como de prácticas de enseñanza innovadoras y variadas para que todos los/as estudiantes tengan garantizado su derecho a una educación de calidad, equitativa e inclusiva. El regreso gradual a las escuelas como las nuevas oportunidades que ofrece lo capitalizado en la enseñanza remota de emergencia podrán ser parte de una educación transformada y transformadora, que ya no será la misma de antes.

\section{Referencias bibliográficas}

Abramowski, A. L. (2012). Los afectos docentes en las relaciones pedagógicas: tensiones entre querer y enseñar. LASA: San Francisco, California May 23-26. Disponible en file://C:/ Users/eugey/Downloads/Los_afectos_docentes_en_las_relaciones_p.pdf

Alasuutari, H. (2020). Abordando la desigualdad en la educación durante y después del COVID-19. Los retos de la educación inclusiva. Education for Global Development, Banco Mundial Blogs. Disponible en https://blogs.worldbank.org/es/education/abordando-la-desigualdad-en-la-educacion-durante-y-despues-del-covid-19-los-retos-de-la

Dussel, I. (2020). Tu escuela en casa: tres aportes clave. Artículos y reseñas para pensar la escuela hoy. Ministerio de Educación Provincia de Córdoba. Disponible en https://tuescuelaencasa.isep-cba.edu.ar/institucional/entre-colegas-docentes/

Echeita, G. y Ainscow, M. (2011). La educación inclusiva como derecho. Marco de referencia y pautas de acción para el desarrollo de una revolución pendiente. Tejuelo, 12, 26-46. Disponible en https://dialnet.unirioja.es/servlet/articulo?codigo=3736956

Gobierno de la República Argentina. (2006). Ley Nacional de Educación.

Gobierno de la República Argentina. (2016). Resolución del Consejo Federal de Educación $n .^{\circ} 311 / 2016$.

Gobierno de la República Argentina. Ministerio de Educación. (2020). Evaluación Nacional del proceso de continuidad pedagógica: la barrera tecnológica fue la principal dificultad para la comunicación de docentes y estudiantes. Disponible en: https://www. argentina.gob.ar/noticias/evaluacion-nacional-del-proceso-de-continuidad-pedagogicala-barrera-tecnologica-fue-la

Hodget, C., Moore, S. et al. (2020). The difference between emergency remote teaching and online learning. Disponible en https://er.educause.edu/articles/2020/3/the-differencebetween-emergency-remote-teaching-and-online-learning

Organización de las Naciones Unidas. (2006). Convención Internacional de los Derechos de la Persona con Discapacidad. Disponible en https://www.un.org/esa/socdev/enable

Organización de las Naciones Unidas. (2015). Agenda 2030 para el Desarrollo Sostenible. Disponible en https://www.un.org/sustainabledevelopment/es/2015/09/la-asamblea-general-adopta-la-agenda-2030-para-el-desarrollo-sostenible/

Organización de las Naciones Unidas. (2016). Convención sobre los Derechos de las Personas con Discapacidad. Comité sobre los Derechos de las Personas con Discapacidad. Observación General n. ${ }^{\circ}$ 4. Artículo 24: Derecho a la educación inclusiva. Disponible en https://rededucacioninclusiva.org/situacion-en-la-region/informes-y-documentos/ comentario-general-n-4-sobre-el-derecho-a-la-educacion-inclusiva/ 
Unesco. (2020). Con uno de cada cinco alumnos privados de escuela, la UNESCO moviliza a los ministros de educación para hacer frente a la crisis por el COVID-19. Disponible en https://www.iesalc.unesco.org/2020/03/10/con-uno-de-cada-cinco-alumnos-privadosde-escuela-la-unesco-moviliza-a-los-ministros-de-educacion-para-hacer-frente-a-la-crisis-por-el-covid-19/

Yadarola, M. E. (2016a). Diseño universal de la enseñanza para una educación inclusiva. Revista Virtual RedEs (Universidad de Cartagena), 3, 26-41. Disponible en http://ojs.udc. edu.co/index.php/redes/article/view/732

YADAROLA, M. E. (2016b). Educación inclusiva como parte de un proyecto inclusivo de vida. En Libro de Actas en CD del IV Congreso Iberoamericano sobre el síndrome de Down. Salamanca. Disponible en http://cddown-inico.usal.es/docs/116.pdf 